\title{
PLANTS
}

\section{FLORA OF THE BESNARD LAKE AREA, NORTH-CENTRAL SASKATCHEWAN}

VERNON L. HARMS, W. P. Fraser Herbarium, University of Saskatchewan, Saskatoon, Saskatchewan, S7N OWO, and ANNA LEIGHTON, 328

Saskatchewan Crescent West, Saskatoon, Saskatchewan. S7M OA4

Introduction Besnard Lake in northcentral Saskatchewan is located about $20-30 \mathrm{~km}$ south of the Churchill River, $60 \mathrm{~km}$ west-northwest of LaRonge, and $40 \mathrm{~km}$ east-southeast of Pinehouse Lake Village, at between latitudes $55^{\circ} 17^{\prime}$ to $31^{\prime} \mathrm{N}$, and longitudes $105^{\circ} 47^{\prime}$ to $106^{\circ} 17^{\prime} \mathrm{W}$. (See Maps 1 and 2.) The area is accessible via the Besnard Lake Road
(Hwy. 910) extending north from Hwy. 165 and crossing the Narrows, and the Morning-Clam Lake Road, forking east from the former to skirt Besnard Lake's south side.

Physiographically, Besnard Lake occurs at the southern border of the Canadian Precambrian Shield, witi the shield landforms north of the lake

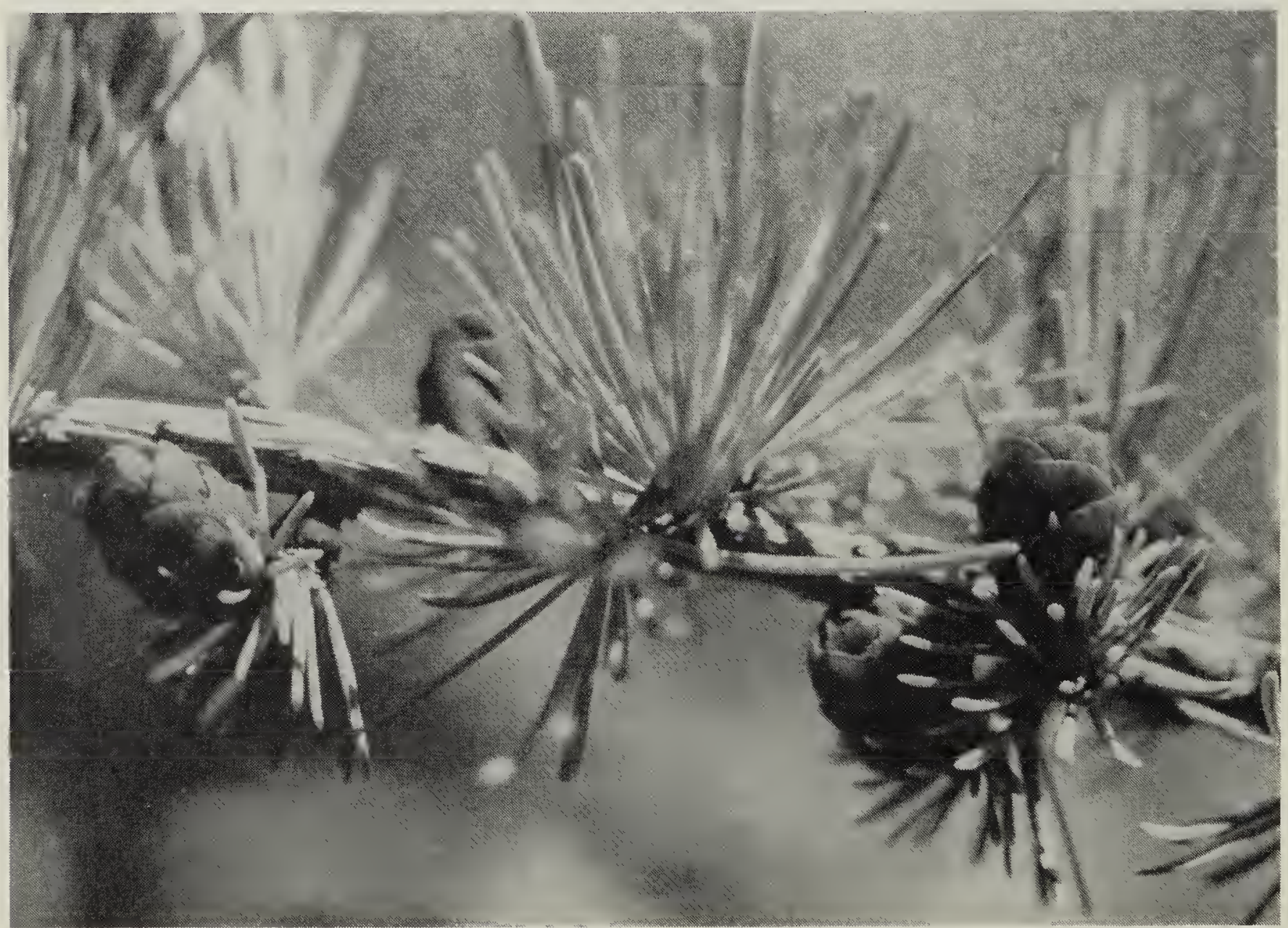




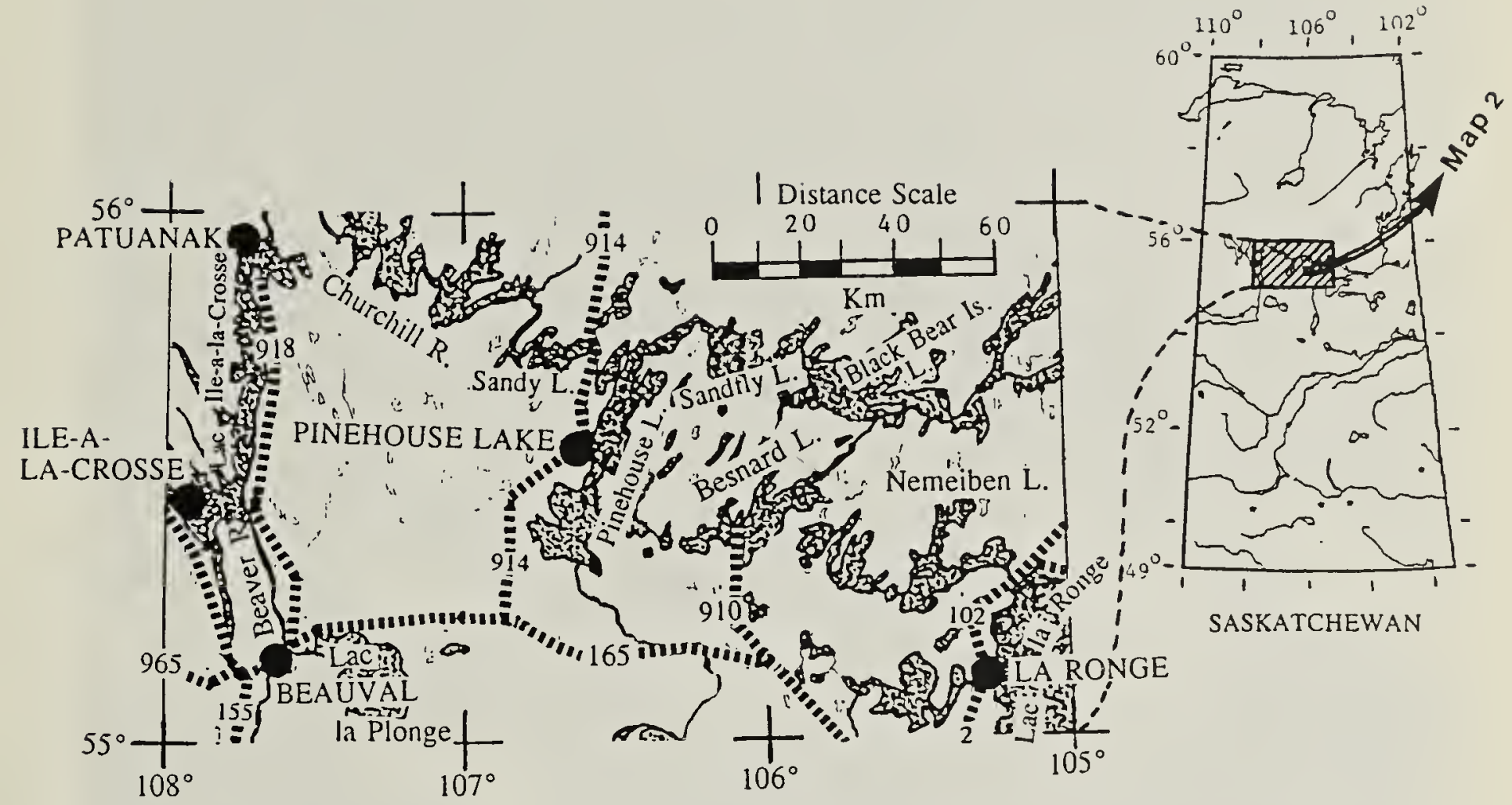

\section{Map 1. LOCATION OF BESNARD LAKE IN NORTH-CENTRAL SASKATCHEWAN.}

characterized by numerous granitic rock outcroppings and shallow soil, and the non-shield landforms south of Besnard Lake characterized largely by sandy, lacustrine or till soils, except locally where organic peaty substrates have developed under bogs and fens. This general area of low relief below the 1500 -foot contour between the Precambrian Shield to the north and the Saskatchewan Uplands to the south was considered distinctive enough to be separately recognized by Rowe as the Upper Churchill Section (\#B.20) ${ }^{2}$ It is situated between the Northern Coniferous (\#B.22) and the Mixedwood (\#B.18a) sections of the Boreal Forest Region of Canada. ${ }^{2}$ It was occupied by the post-glacial Lake Hyper-Churchill, resulting in the generally flat to undulating surfaces now present. Apparently, the sandy lacustrine and till deposits were originally derived from thin basal sandstone on the Precambrian basement to the north. ${ }^{2}$
The original Jack Pine forests that predominated on the sandy upland plains and ridges in the Besnard Lake area have been destroyed to a large extent by extensive forestry clearcutting. The lower, moister sites are largely characterized by Black Spruce woods, often admixed with some White Spruce. Limited stands of aspen-dominated deciduous woods and mixedwoods of various combinations of all the aforementioned tree species, plus also Balsam Poplar, White Birch and River Alder, exist mainly near lake shores and some stream margins. Balsam Fir is uncommon and occurs mostly as small, understorey shrubs less than $1 \mathrm{~m}$ high in the mixed and spruce woods. Some Tamarack occur with Black Spruce in most treed bogs. Open bogs and fens characterize low, wet, poorly drained depressions.

Besnard Lake is noted for its Bald Eagle nesting sites. ${ }^{1}$ With the present 


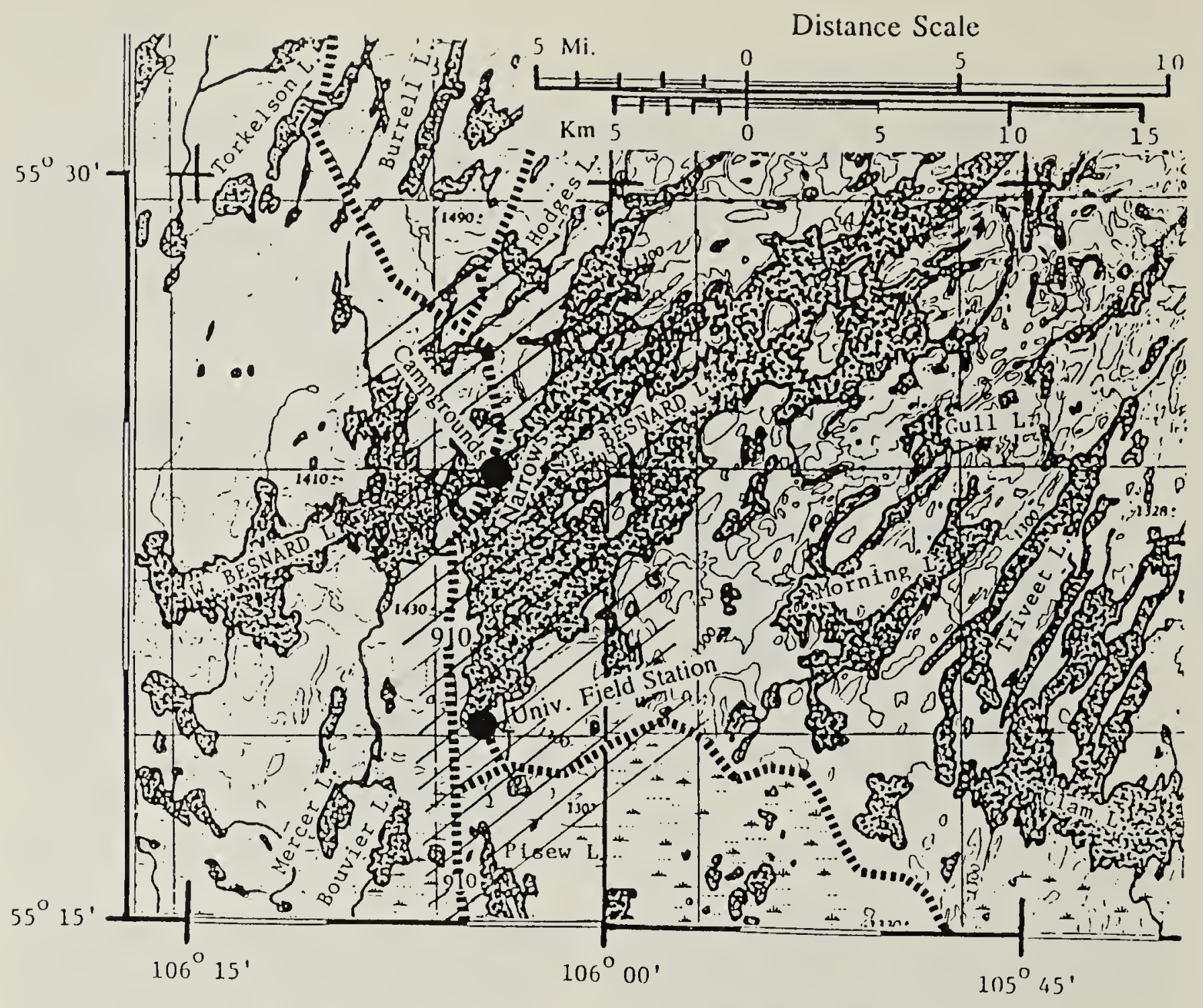

Map 2. BESNARD LAKE AND VICINITY.

location there of a University of Saskatchewan research station under the direction of Dr. Gary R. Bortolotti, it has also become an important study area for other wildlife, especially American Kestrels and other birds of prey, and their rodent prey species.

The following list of 210 species of higher (i.e., vascular) plants (Table 1) was compiled by the authors, based primarily on plant collections made in the general Besnard Lake vicinity by V. L. Harms in June 1992 and by Anna Leighton in 1977-78, but with these supplemented by the 1978 collections of Jon, Naomi and Peter Gerrard, Doug Whitfield and Stacy Tessaro. Despite the wider coordinates given earlier for the general Besnard Lake area, almost all plant collections were made in the Narrows region, on the central islands, and along the south side, between longitudes $105^{\circ} 57^{\prime}$ and $106^{\circ} 05^{\prime} W$ (see hatched area on Map 2).

The 210 species of vascular plants recorded in the overall area are grouped into 134 genera and 58 families. They include 15 species of pteridophytes (i.e., ferns and fernallies), 6 species of coniferous gymnosperms, and 189 species of angiosperms (i.e., flowering plants). Less than $4 \%$ (i.e., only 8 of 210 species) represent introduced plants (i.e., either invasive weeds or escaped cultigens), which are indicated by asterisks in the subsequent list. The following three plants recorded from the Besnard Lake area are considered rare in Saskatchewan: Poa arctica (Arctic Bluegrass), Polygonum punctatum var. confertiflorum (Dotted Water Smartweed) and Lilium philadelphium var. andinum 


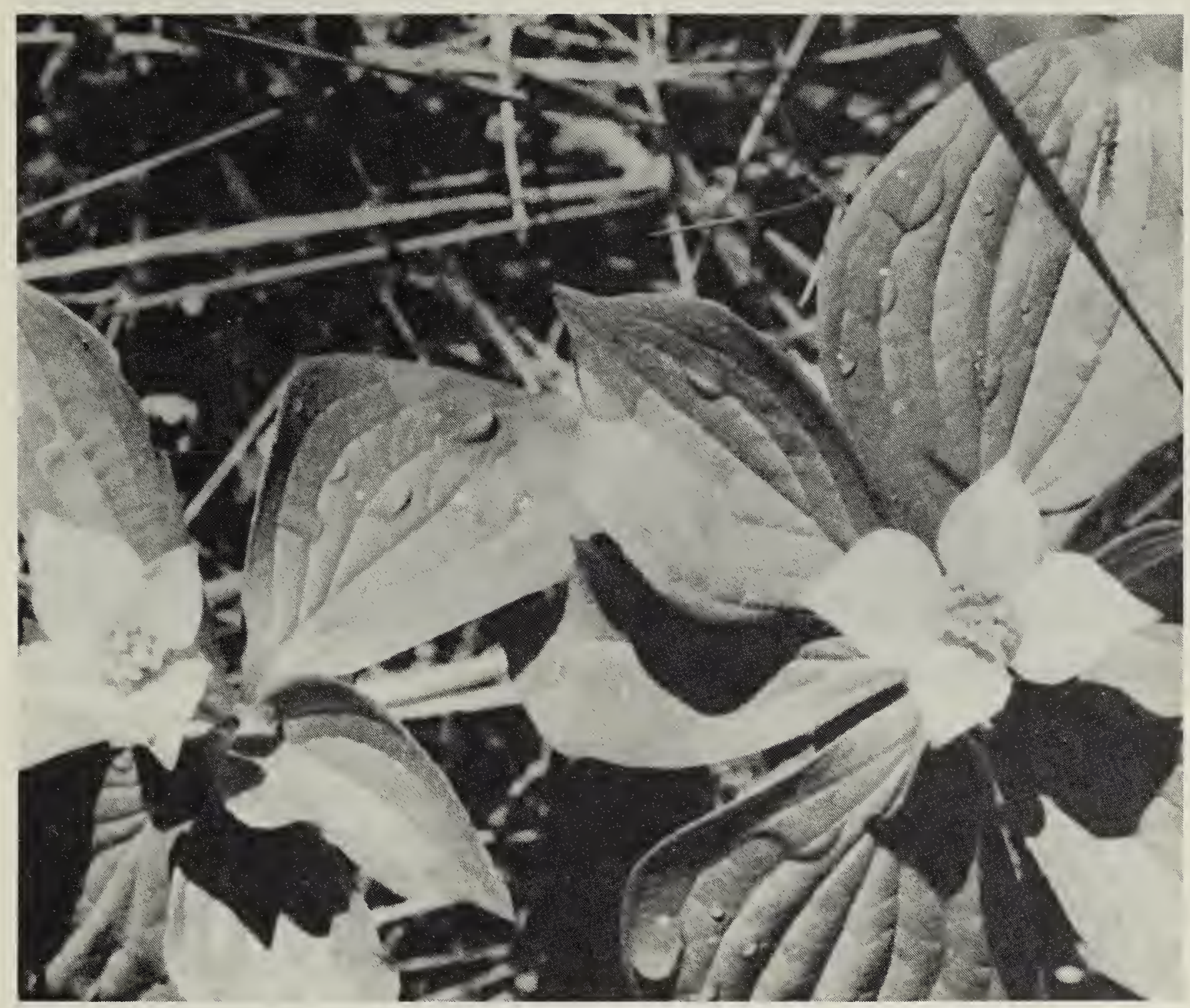

Bunch Berry (Cornis canadensis L.)

Frank A. Switzer

forma immaculata (Immaculate Yellow Lily). Voucher specimens documenting all listed plants are filed in the W.P. Fraser Herbarium, at the University of Saskatchewan, Saskatoon, Saskatchewan.

This species inventory list should not be considered a fait accompli, but rather a starting point for the finding of additional plant species records from this general area. Conceivably, about 100 more vascular plants might well be expected for an area of this size at this latitude.

Acknowledgements Appreciation is due to Doug Whitfield who hired the junior author as a field assistant in 1977-78, and to the Boreal Institute of the University of Alberta for sup- porting the former's Bald Eagle studies at Besnard Lake. The voluntary contributions of the Gerrard family, as indicated earlier, are thankfully noted. The senior author's collecting time at Besnard Lake in June 1992 occurred while he was involved with a Boreal Forest Ecology Field Camp sponsored by the University of Saskatchewan Extension Division under the direction of Peter Jonker.

1. GERRARD, J.M. and G.R. BORTOLOTTI. 1988. The Bald Eagle haunts and habitats of a wilderness monarch. Smithsonian Institution, Washington, DC. 177 pp. (Paperback copy published by Western Producer, Saskatoon, SK.)

2. ROWE, J.S. 1972. The forest regions of Canada. Canadian Forestry Service Publ. No. 1300. Ottawa, ON. 172 pp. 


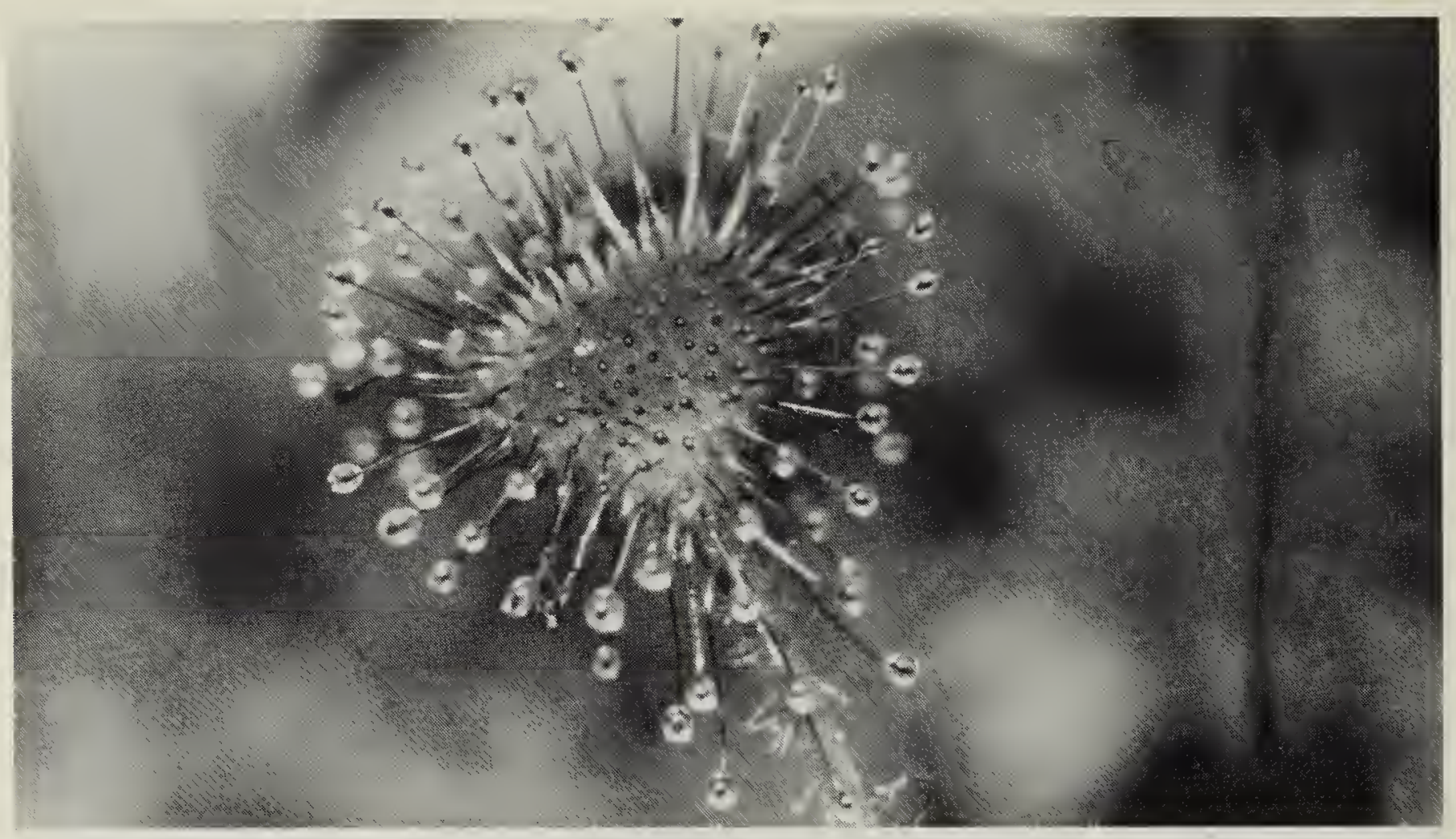

Round-leaved Sundew (Drosera rotundifola L.)

Gary W. Seib

\section{Table 1. VASCULAR PLANTS RECORDED AND DOCUMENTED} IN THE BESNARD LAKE VICINITY.

* = introduced (i.e., non-native) plants; $(R)=$ plants rare in Saskatchewan

ADIANTACEAE (Maidenhair Family) Cryptogramma crispa (L.) R.Br. var. acrostichoides (R.Br.) Clarke Parsley Fern

ALISMATACEAE (Water-plantain Family) Sagittaria cuneata Sheld. - Arumleaved Arrowhead

APIACEAE (Parsley or Carrot Family)

Cicuta maculata L. var. angustifolia Hook. - Western Water Hemlock Cicuta mackenzieana Raup. - Water Hemlock

Sium suave Walt. - Water Parsnip APOCYNACEAE (Dogbane Family) Apocynum androsaemifolium L. -

Spreading Dogbane

ARACEAE (Arum Family) Acorus calamus L. - Sweet Flag Calla palustris L. - Marsh Calla; Wild Arum; Wild Calla-lily

ARALIACEAE (Ginseng Family) Aralia hispida Vent. - Bristly
Sarsaparilla

Aralia nudicaulis L. - Wild

Sarsaparilla

ASPLENIACEAE (Spleenwort

Family)

Dryopteris carthusiana (Vill.) H.P.

Fuchs - Narrow Spinulose Shield Fern

Dryopteris fragrans (L.) Schott. -

Fragrant Shield Fern

Gymnocarpium dryopteris (L.)

Newm. - Oak Fern

Woodsia ilvensis (L.) R. Br. - Rusty

Woodsia

ASTERACEAE (Aster or Composite Family)

Achillea millefolium L. ssp. borealis

(Bong.) Breit. - Northern Yarrow Achillea millefolium L. ssp. borealis

(Bong.) Breit. f. rhodantha

(LePage) Scoggan - Pink-

flowered Northern Yarrow

Achillea millefolium L. ssp. lanulosa

(Nutt.) Piper - Common Yarrow Antennaria parvifolia Nutt. - Low or

Small Pussytoes or Everlasting Aster ciliolatus Lindl. - Lindley's Blue Aster 
Bidens cernua L. - Smooth Beggarticks

Chrysanthemum leucanthemum L. Oxeye Daisy*

Crepis tectorum L. - Narrow-leaved Hawksbeard*

Erigeron philadelphicus L. -

Philadelphia Fleabane

Hieracium umbellatum L. - Canada Hawkweed

Matricaria matricarioides (Less.)

Porter - Pineapple Weed*

Petasites palmatus (Ait.) A. Gray -

Palmate-leaved Colt's-foot

Senecio indecorus Greene -

Unsightly Groundsel

Senecio pauperculus Michx. -

Depauperate Groundsel

Solidago canadensis L. var. salebrosa (Piper) M.E. Jones -

Canada Goldenrod

Solidago hispida Muhl. - Shaggy

Goldenrod

Solidago spathulata DC. var. neoalaskana (Gray) Cronq. Mountain Goldenrod

BALSAMINACEAE (Jewelweed

Family)

Impatiens capensis Meerb. -

Spotted Touch-me-not

BETULACEAE (Birch Family)

Alnus crispa (Ait.) Pursh - Green

Alder

Alnus rugosa (Du Roi) Spreng. -

Speckled or River Alder

Betula neoalaskana Sarg. - Alaska

White or Paper Birch

Betula pumila L. var. glandulifera

Regel - Glandular Shrub Birch

BORAGINACEAE (Borage Family)

Mertensia paniculata (Ait.) G. Don -

Tall Lungwort

BRASSICACEAE (Mustard Family)

Arabis divaricarpa A. Nels. var.

stenocarpa M. Hopkins - Purple

Rock Cress

Arabis hirsuta (L.) Scop. var.

glabrata T\&G - Hirsute Rock

Cress

Lepidium densiflorum Schrad. -
Common Pepper-grass*

Sisymbrium loeselii L. - Tall Hedge Mustard*

CAMPANULACEAE (Bluebell

Family)

Campanula aparinoides Pursh -

Marsh Bellflower

Campanula rotundifolia L. -

Common Harebell or Bluebell

CAPRIFOLIACEAE (Honeysuckle

Family)

Linnaea borealis L. - Twin-flower

Lonicera dioica L. var. glaucescens

(Rydb.) Butters - Twining

Honeysuckle

Viburnum edule (Michx.) Raf. - Low

Bush Cranberry

CARYOPHYLLACEAE (Pink

Family)

Moehringia lateriflora (L.) Fenzl. -

Grove Sandwort

Stellaria calycantha (Ledeb.) Bong. -

Northern Chickweed

Stellaria longifolia Muhl. - Longleaved Stitchwort, Starwort or Chickweed

Stellaria longipes Goldie - Longstalked Chickweed

CHENOPODIACEAE (Goosefoot

Family)

Chenopodium capitatum (L.)

Aschers - Strawberry Blite

CORNACEAE (Dogwood Family)

Cornus canadensis L. - Bunch

Berry; Dwarf Dogwood

CUPRESSACEAE (Cypress Family) Juniperus communis L. - Low or

Ground Juniper

CYPERACEAE (Sedge Family)

Carex cf. adusta Boott. - Browned Sedge

Carex cf. aenea Fern - Hay Sedge

Carex aquatilis Wahl. - Water Sedge

Carex atherodes Spreng. - Awned

Sedge

Carex aurea Nutt. - Golden Sedge

Carex crawfordii Fern - Crawford's

Sedge 
Carex disperma Dewey - Softleaved or Two-seeded Sedge

Carex houghtonii Torr. - Houghton's Sedge

Carex cf. lanuginosa Michx. - Woolly Sedge

Carex rostrata Stokes - Beaked

Sedge

Carex stipata Muhl. - Awl-fruited

Sedge

Eleocharis palustris (L.) R. \& S. -

Creeping Spike-rush

Eriophorum angustifolium Honck. -

Tall Cotton-grass

Eriophorum brachyantherum Trautv.

- Close-sheathed Cotton-grass

Eriophorum chamissonis C.A. Mey.

- Chamisso's Cotton-grass

Eriophorum gracile K. Koch -

Slender Cotton-grass

Eriophorum vaginatum $\mathrm{L}$. -

Sheathed Cotton-grass

Scirpus cyperinus (L.) Kunth -

Wool-grass

Scirpus hudsonianus (Michx.) Fern.

- Alpine Cotton-grass

Scirpus microcarpus Presl. -

Small-fruited Sedge

DROSERACEAE (Sundew Family)

Drosera anglica Huds. -

Oblong-leaved Sundew

Drosera rotundifolia L. -

Round-leaved Sundew

EQUISETACEAE (Horsetail Family)

Equisetum arvense L. - Common or

Field Horsetail

Equisetum fluviatile L. - Swamp or

Water Horsetail

Equisetum scirpoides Michx. - Dwarf

Scouring-rush; Sedge-like

Horsetail

Equisetum sylvaticum L. - Woodland

Horsetail

Equisetum variegatum Schleich. -

Variegated Horsetail

ERICACEAE (Heath Family)

Andromeda polifolia L.. - Bog

Rosemary

Arctostaphylos uva-ursi (L.) Spreng.

- Common Bearberry;

Kinnikinnick
Chamaedaphne calyculata (L.)

Moench. - Leatherleaf

Kalmia polifolia Wang. - Pale or Bog

Laurel

Ledum groenlandicum Oeder -

Common Labrador-tea

Oxycoccus microcarpus Turcz. -

Small Bog or Swamp Cranberry

Oxycoccus quadripetalus Gilib. -

Larger Bog or Swamp Cranberry

Vaccinium myrtilloides Michx. -

Canada Blueberry

Vaccinium vitis-idaea L. -

Lingenberry; Dry-ground,

Mountain, Low or Bog Cranberry;

Cowberry

FABACEAE (Legume or Pea

Family)

Astragalus canadensis L. - Canada

Milk Vetch

Caragana arborescens Lam. -

Caragana; Siberian Pea-shrub*

Lathyrus ochroleucus Hook. -

Cream-colored Pea-vine or

Vetchling

Trifolium repens L. - White Clover*

Vicia americana Muhl. - American

Vetch

FUMARIACEAE (Fumitory Family)

Corydalis aurea Willd. - Golden

Corydalis

Corydalis sempervirens (L.) Pers. -

Pink or Pale Corydalis

GENTIANACEAE (Gentian Family)

Gentiana amarella L. - Northern

Gentian

GERANIACEAE (Geranium Family) Geranium bicknellii Britt. - Bicknell's

Wild Geranium

GROSSULARIACEAE (Currant

Family)

Ribes glandulosum Grauer - Skunk Currant

Ribes hudsonianum Richards. -

Northern Black Currant

Ribes oxyacanthoides L. S. lato -

Northern or Canada Gooseberry

HALORAGIDACEAE (Water-milfoil

Family)

Myriophyllum exalbescens Fern. -

American Water-milfoil 
HYDROPHYLLACEAE (Water-leaf

Family)

Phacelia franklinii (R. Br.) A. Gray -

Franklin's Scorpion-weed

HYPERICACEAE (St. John's-wort

Family)

Hypericum majus (Gray) Britt. -

Large Canada St. John's-wort

JUNCACEAE (Rush Family)

Juncus alpinus Vill. var. rariflorus

Hartm. - Alpine Rush

Juncus dudleyi Wieg. - Dudley's

Path Rush

LAMIACEAE (Mint Family)

Lycopus uniflorus Michx. - Northern

Water-horehound

Mentha arvensis L. - Wild or Field

Mint

Stachys palustris L. var. pilosa

(Nutt.) Fern. - Marsh Hedge-

nettle

LENTIBULARIACEAE (Bladderwort

Family)

Utricularia intermedia Hayne -

Flat-leaved Bladderwort

LILIACEAE (Lily Family)

Lilium philadelphicum L. var. andinum (Nutt.) Ker. - Western

Red Wood Lily

Lilium philadelphicum L. var.

andinum (Nutt.) Ker forma

immaculatum Raup - Immaculate

Yellow Lily. (R)

Maianthemum canadense Desf. var. interius Fern. - Two-leaved

Solomon's-seal

Smilacina trifolia (L.) Desf. -

Three-leaved Solomon's-seal

LORANTHACEAE (Mistletoe

Family)

Arceuthobium americanum Nutt. -

Pine or American Mistletoe

LYCOPODIACEAE (Club-moss

Family)

Lycopodium annotinum L. - Stiff

Club-moss

Lycopodium clavatum L. - Running

Club-moss

Lycopodium complanatum L. -
Ground-cedar; Trailing Club-

moss

Lycopodium dendroideum Michx. -

Ground-pine; Tree Club-moss

MYRICACEAE (Bayberry Family)

Myrica gale L. - Sweet Gale;

Bog-myrtle

NYMPHACEAE (Water Lily Family) Nuphar variegatum Engelm. -

Yellow Pond-lily

ONAGRACEAE (Evening-primrose

Family)

Circaea alpina L. - Small

Enchanter's-nightshade

Epilobium angustifolium L. -

Fireweed

Epilobium ciliatum Raf. - Northern

Willow-herb

Epilobium palustre L. - Marsh

Willow-herb

ORCHIDACEAE (Orchid Family)

Cypripedium acaule Ait. - Stemless

Lady's-slipper

Cypripedium calceolus L. var.

parviflorum (Salisb.) Fern. - Small

Yellow Lady's-slipper

Goodyera repens (L.) R. Br. -

Lesser Rattlesnake Plantain

Platanthera hyperborea (L.) Lindl. -

Northern Green Bog Orchid

Platanthera obtusata (Banks) Lindl.

- Small Northern Bog Orchid

Spiranthes romanzoffiana Cham. \&

Schl. - Hooded Ladies'-tresses

PINACEAE (Pine Family)

Abies balsamea (L.) Mill. - Balsam

Fir

Larix laricina (Du Roi) K. Koch -

Tamarack; American Larch

Picea glauca (Moench) Voss - White

Spruce

Picea mariana (Mill.) BSP. - Black

Spruce

Pinus banksiana Lamb. - Jack Pine

PLANTAGINACEAE (Plantain

Family)

Plantago major L. - Common or

Broadleaf Plantain; White-man's

Foot $^{\star}$ 
POACEAE (Grass Family)

Agropyron trachycaulum (Link)

Malte - Slender or Western

Wheatgrass

Agrostis scabra Willd. - Rough Hairgrass; Tickle-grass

Alopecurus aequalis Sobol. - Shortawned or Water Foxtail

Calamagrostis canadensis (Michx.)

Beauv. - Blue Joint; Marsh Reed Grass

Elymus innovatus Beal. - Hairy Wildrye

Glyceria borealis (Nash) Batch. Northern Manna Grass

Festuca saximontana Rydb. - Rocky Mountain Fescue

Hierochloe odorata (L.) Beauv. Sweet Grass

Oryzopsis asperifolia Michx. White-grained Mountain-rice

Oryzopsis pungens (Torr.) Hitch. -

Slender Mountain-rice

Phalaris arundinacea L. - Reed

Canary Grass

Phragmites australis (Cav.) Trin. Common Reed Grass

Poa arctica R.Br. - Arctic Blue Grass. (R)

Poa interior Rydb. - Inland or Woodland Blue Grass

Poa palustris L. - Fowl Blue Grass Schizachne purpurascens (Torr.)

Swallen - Purple Oat-grass

POLYGONACEAE (Buckwheat

Family)

Polygonum amphibium L. var. stipulaceum (Coleman) Fern. var. hirtuosum (Farw.) Fern. - Water or Swamp Persicaria or Smartweed

Polygonum cilinode Michx. - Fringed Black Bindweed

Polygonum punctatum Ell. var. confertiflorum (Meisn.) Fassett Dotted Water Smartweed. (R) Rumex triangulivalvis (Dans.) Rech.

$\mathrm{f}$. - Willow-leaved or Mexican Dock

POLYPODIACEAE (Polypody

Family)
Polypodium virginianum $\mathrm{L}$. Common or Virginia Polypody; Common Rock-tripe

\section{POTAMOGETONACEAE}

(Pondweed Family)

Potamogeton gramineus L. - Grassleaved Pondweed

Potamogeton natans L. - Common Floating Pondweed

Potamogeton pusillus L. - Small Pondweed

Potamogeton richardsonii (Benn.) Rydb

Potamogeton zosteriformis Fern. Eel-grass Pondweed

PRIMULACEAE (Primrose Family)

Lysimachia thyrsiflora L. - Tufted Loosestrife

Trientalis borealis Raf. - Northern Star Flower

PYROLACEAE (Wintergreen

Family)

Chimaphila umbellata (L.) Bart. Prince's-pine

Moneses uniflora (L.) A. Gray - Oneflowered Wintergreen

Orthilia secunda (L.) House - Onesided Wintergreen or Pyrola Pyrola asarifolia Michx. - Pink Wintergreen or Pyrola

Pyrola elliptica Nutt. - Common Shinleaf

Pyrola virens Schweig. - Greenflowered Wintergreen or Pyrola

RANUNCULACEAE (Buttercup Family)

Actaea rubra (Ait.) Willd. forma neglecta (Gilman) Robins - White Baneberry

Caltha natans Pall. - Floating Marsh Marigold

Caltha palustris L. - Marsh-marigold Pulsatilla ludoviciana (Nutt.) Heller -

Crocus Anemone; Pasque-flower; Prairie-crocus

Ranunculus flammula L. - Creeping Spearwort

Ranunculus gmelinii DC. - Small

Yellow Watercrowfoot

Ranunculus lapponicus L. - Lapland 
Buttercup

Ranunculus pensylvanicus L. f. -

Bristly Buttercup

ROSACEAE (Rose Family)

Amelanchier alnifolia Nutt. -

Saskatoon Serviceberry

Fragaria glauca (S. Wats.) Rydb. -

Smooth Wild Strawberry

Geum aleppicum Jacq. - Yellow

Avens

Potentilla norvegica L. - Rough

Cinquefoil

Potentilla palustris (L.) Scop. -

Marsh Cinquefoil

Potentilla pensylvanica L. var.

pensylvanica - Prairie or

Pennsylvania Cinquefoil

Prunus pensylvanica L. f. - Pin

Cherry; Bird Cherry

Rosa acicularis Lindl. ssp. sayi

(Schwein.) Lewis - Prickly Wild

Rose

Rosa woodsii Lindl. - Wood's Wild

Rose

Rubus idaeus L. var. strigosus

(Michx.) Maxim. - Wild Red or

Canada Raspberry

Rubus pubescens Raf. - Dewberry;

Bramble; Running Raspberry

RUBIACEAE (Madder Family)

Galium boreale L. - Northern

Bedstraw

Galium trifidum L. - Small Bedstraw Galium triflorum Michx. -

Sweet-scented Bedstraw

SALICACEAE (Willow Family)

Populus balsamifera L. - Balsam or

Black Poplar

Populus tremuloides Michx. -

Trembling Aspen; White Poplar

Salix pedicellaris Anderss. - Bog

Willow

Salix petiolaris J.E. Smith - Basket

Willow

Salix planifolia Pursh - Flat-leaved
Willow

Salix pyrifolia Anderss. - Balsam or

Pear-leaved Willow

SANTALACEAE (Sandalwood

Family)

Comandra umbellata A. DC. var umbellata - Common Comandra; Bastard Toad-flax

Geocaulon lividum (Richards.) Fern.

- Northern Comandra

SAXIFRAGACEAE (Saxifrage

Family)

Heuchera richardsonii R. Br. -

Richardson's Alum-root

Mitella nuda L. - Mitrewort;

Bishop's-cap

Parnassia palustris L. - Northern

Grass-of-Parnassus

Saxifraga tricuspidata Rottb. -

Three-toothed Saxifrage

SCROPHULARIACEAE (Figwort

Family)

Melampyrum lineare Desv. - Cow-

wheat

SPARGANIACEAE (Bur-reed

Family)

Sparganium angustifolium Michx. -

Narrow-leaved Bur-reed

Sparganium eurycarpum Engelm. -

Broad-fruited Bur-reed

TYPHACEAE (Cat-tail Family)

Typha latifolia L. - Cat-tail

VIOLACEAE (Violet Family)

Viola adunca J.E. Smith - Early Blue or Hooked-spur Violet

Viola palustris L. - Marsh Violet

Viola renifolia Gray var. brainerdii

(Greene) Fern. - Kidney-leaved

Violet

(Voucher specimens are filed in the W.P. Fraser Herbarium, University of Saskatchewan, Saskatoon, Saskatchewan.) 